\title{
CHANGING TRENDS OF CANDIDA ISOLATES AND THEIR ANTIFUNGAL SUSCEPTIBILITY PATTERN IN VULVOVAGINAL CANDIDIASIS CASES OF TRIPURA, NORTH EAST INDIA
}

\author{
Jhinuk Basu Mullick1, Tapan Majumdar², Jayanta Ray³, Samir Kumar Sil ${ }^{4}$ \\ ${ }^{1}$ PhD Scholar, Department of Human Physiology, Tripura University, Agartala, Tripura. \\ ${ }^{2}$ Associate Professor, Department of Microbiology, Agartala Government Medical College, Agartala, Tripura. \\ ${ }^{3}$ Associate Professor, Department of Obstetrics and Gynaecology, Agartala Government Medical College, Agartala, Tripura. \\ ${ }^{4}$ Professor, Department of Human Physiology, Tripura University, Agartala, Tripura.
}

\begin{abstract}
BACKGROUND: Candida species are a part of the complex endogenous vaginal microflora, which under pathological condition cause vulvovaginal candidiasis. Worldwide it is the second most common cause of vaginitis after bacterial vaginosis and in India it accounts for $18-20 \%$ of clinical visits.

AIM: To determine the spectrum of Candida species among the subjects of suspected vulvovaginal candidiasis with an objective to isolate and identify all the Candida species and determine their antifungal susceptibility pattern.

SETTINGS AND DESIGN: The study was cross sectional observational, conducted on 275 symptomatic outdoor and admitted patients in the Department of Obstetrics and Gynaecology in a tertiary care hospital in Tripura between August 2012 and April 2015.

MATERIALS AND METHODS: Discharge was collected from patients and identified by Grams staining and wet mount test. Culturing was done in Sabouraud dextrose agar followed by speciation through germ tube test, corn meal agar morphologies and chromogenic identification in HiCrome agar. Antifungal susceptibility was determined through E-test for Polyenes and Azoles.

RESULT: Vulvovaginal candidiasis was found at a frequency of $25 \%$ with non-albicans Candida species (62\%) prevailing over C. albicans (38\%). Highest frequency was observed in the 40-49 age group. Highest susceptibility was seen for fluconazole and least for ketoconazole.

CONCLUSION: The frequency of vulvovaginal candidiasis was found to be higher than other prevalence studies from India. The study also showed increasing shift towards non-albicans Candida species from C. albicans. The reason for high frequency among the menopausal group and ectopic pregnancy cases could be topics for future studies. Amphotericin B and fluconazole were effective towards most species and hence could be used to treat the local population.
\end{abstract}

KEYWORDS: Vulvovaginal Candidiasis, Non-Albicans Candida Species, Antifungal Susceptibility Test.

HOW TO CITE THIS ARTICLE: Jhinuk Basu Mullick, Tapan Majumdar, Jayanta Ray, Samir Kumar Sil. “Changing Trends of Candida Isolates and their Antifungal Susceptibility Pattern in Vulvovaginal Candidiasis Cases of Tripura, North East India." Journal of Evolution of Medical and Dental Sciences 2015; Vol. 4, Issue 94, November 23; Page: 15918-15922,

DOI: $10.14260 /$ jemds/2015/2317.

INTRODUCTION: Candida species are a part of the complex endogenous vaginal microflora; however, under morphogenesis inducing conditions their number increases rapidly causing profuse 'cottage cheese like' discharge, pruritus and frequent and uncontrollable micturition.

This pathological condition is known as vulvovaginal candidiasis (VVC); 75\% of women are reported to experience at least one episode of VVC in their lifetime.[1]

Financial or Other, Competing Interest: Mullick JB reports grants from Department of Biotechnology (DBT) under Ministry of Science and Technology, India, during the conduct of the study; non-financial support from Dr. Sumathi Muralidhar and from Dr. KVR Reddy, outside the submitted work. This study was part of a larger study of incidence of sexually transmitted infections in the state of Tripura, the project was entitled Isolation and Characterization of pathogens associated with Sexually Transmitted Infections and in vitro testing of microbicide, SSALF24..

Submission 02-11-2015, Peer Review 03-11-2015,

Acceptance 13-11-2015, Published 20-11-2015.

Corresponding Author:

Jhinuk Basu Mullick,

Department of Human Physiology,

Tripura University, Suryamaninagar,

Agartala-799022, Tripura.

E-mail: jhinuk.bm@gmail.com

DOI:10.14260/jemds/2015/2317.
Worldwide it is the second most common cause of vaginitis after bacterial vaginosis accounting for approximately $40 \%$ of cases. ${ }^{[2]}$ In India, it accounts for $18-20 \%$ of clinical visits. ${ }^{[3]}$

Risk factors include use of oral contraceptive pills, young age at first sexual encounter, intercourse more than four times per month, diabetes, pregnancy, recent antibiotic use and HIV/AIDS.

In the last two decades a major shift has been observed from Candida albicans to non-albicans Candida (NAC) species causing $35-65 \%$ of all candidal infections.[4] A possible explanation for more frequent isolation of NAC species from vulvovaginitis patients may be the increased use of topical antifungal agents available as over-the-counter preparations, resulting in most non-albicans Candida species showing higher MICs and therefore difficulty in treatment.

To prevent NAC spp. from exerting selection pressure towards resistance by empirical anti-fungal treatment or prophylaxis, speciation of Candida isolates is essential prior to antifungal susceptibility testing. North East India is known to have higher prevalence of HIV and presence of sexually transmitted infections (STIs) can increase the co-infection of HIV by two to five fold.[5] Study in 2009 from Assam.[6] showed VVC $(21.5 \%)$ to be the most common finding amongst STIs. Lack of study from Tripura, also situated in 
North East India, compounded with the high prevalence of HIV in the North East encouraged us to research on the topic.

MATERIALS AND METHODS: The study was cross-sectional observation, conducted in a tertiary hospital in Tripura between August 2012 and April 2015 post clearance from institutional ethical committee. Study subjects comprised of excessive vaginal or penile discharge, cases with genital pruritus or frequent micturition within the reproductive age of 18-49 years. Those on concurrent antibiotic use, menstruating, pregnant, or with any intrauterine device were excluded from the study. After obtaining informed consent, details were recorded in a structured questionnaire about the subject's demographic profile, routine hygiene practices and sexual history. Discharge was collected using cotton swabs from male and female subjects in duplicate.

Candida Species Identification and Culture: Direct Grams stained smears were observed for presence of fungal elements. Wet mount was done with $10 \%$ potassium hydroxide. Discharge samples were cultured in Sabouraud dextrose agar (SDA) with and without chlorhexidine. Yeast identification was done through colony characteristics, Grams stain reaction and sugar assimilation test. Further, Candida speciation was confirmed by germ tube test and cultures in Corn-meal agar (CMA) and HiCrome agar (HCA) (HiMedia laboratories, Mumbai, India).

Antifungal Susceptibility Testing (AFST): AFST was assessed using E test format according to the guidelines of the Clinical and Laboratory Standards Institute.[7] for amphotericin B, fluconazole, ketoconazole and voriconazole. In brief, inoculum was prepared in $5 \mathrm{ml}$ of sterile $0.9 \%$ saline from overnight grown fresh culture in SDA to a turbidity corresponding to 0.5 McFarland standards (Equivalent to $10 .^{8}$ cells $/ \mathrm{ml}$ ). Mueller-Hinton agar with $2 \%$ glucose and $0.5 \mu \mathrm{g} / \mathrm{ml}$ methylene blue was used for the test to which E-strip (HiMedia laboratories, Mumbai, India) for each antifungal agent was applied and read at $24 \mathrm{~h}$. Susceptibility interpretation was done as per CLSI guidelines.[7] Strains of C.

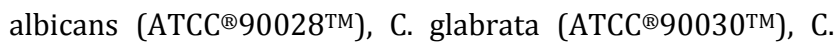
krusei (ATCC ${ }^{\circledR} 6258^{\mathrm{TM}}$ ) C. tropicalis (ATCC ${ }^{\circledR} 750^{\mathrm{TM}}$ ) and C. parapsilosis (ATCC ${ }^{\circledR 22019}{ }^{\mathrm{TM}}$ ) were used as quality controls.

RESULT: A total of 275 symptomatic cases were sampled of which VVC was confirmed through presence of ample fungal elements in Gram's stains of direct smears in 70 (25\%) subjects. Out of the 70 cases, 58 (Cultural sensitivity 83\%) could be isolated by culture. Frequencies of various Candida spp. identified are as shown in Fig 1. and method of speciation in HCA is as shown in Fig 2. C. albicans constituted of $22(38 \%)$ isolates and the rest 36 (62\%) non-albicans included 15C. tropicalis, 12C. glabrata, 7C. krusei, 1C. parapsilosis and $1 \mathrm{C}$. guilliermondii. A single case was found in a male subject belonging to $\mathrm{C}$. albicans.

Highest numbers of Candida spp. were isolated from the 40-49 age group. Species wise too C. albicans, C. glabrata and C. krusei showed a gradual increase reaching a maximum in the 40-49 years. However, C. tropicalis peaked in occurrence in the 30-39 age group and decreased drastically for the 40-
49 age group. Distribution of different Candida spp. among the age groups of study subjects are shown in Fig 3. Out of the 58 diagnosed cases, belonging to various age groups were 11 subjects of post-operative diagnosed ectopic pregnancy. Predominant isolates among such subjects were C. albicans and C. tropicalis.

Antifungal sensitivity was tested against fluconazole, voriconazole, ketoconazole and amphotericin $\mathrm{B}$ and the results can be observed in Table 1 . Fluconazole sensitivity was the highest with overall susceptibility being $88 \%$. Voriconazole susceptibility was also found to be high at $84 \%$ among all the isolates. Ketoconazole susceptibility was seen mostly in C. albicans (82\%), and to some degree in C. tropicalis $(67 \%)$ isolates, while no $C$. glabrata isolate was susceptible. Among the fluconazole resistant isolates, all the C. tropicalis isolates that were fluconazole resistant were also resistant to voriconazole; however, two isolates of C. glabrata and the only isolate of $\mathrm{C}$. krusei showed sensitivity to voriconazole even at low MIC (0.38) value.

All fluconazole resistant isolates were resistant to high MICs (>32) of ketoconazole. The overall susceptibility to amphotericin B was $81 \%$. Susceptibility was seen mostly among the C. albicans (91\%) and C. krusei (100\%) isolates, while only $60 \%$ of C. tropicalis isolates were susceptible. C. glabrata isolates were susceptible with higher MIC values. The only occurring C. parapsilosis isolate was resistant to ketoconazole and amphotericin $\mathrm{B}$ and sensitive to the rest of the azoles. C. guilliermondii isolate was sensitive to all the drugs tested.

DISCUSSION: VVC rates were found to be $25 \%$ in the present study, which is higher than the prevalent range of $18 \%-20 \%$ published in other studies from India. ${ }^{[3,8]}$ Lack of awareness for routine hygienic practices, poor dietary habits, and use of over-the-counter antibiotic and antifungal drugs were considered to be important factors for higher prevalence among the study subjects. The incidence of candidiasis due to NAC spp. is increasing.[9] Isolation of $62 \%$ of NAC spp. in the current study over $38 \%$ of C. albicans was in agreement with the scenario. Among the NAC spp., the highest frequency was of C. tropicalis (26\%) followed by C. glabrata (21\%) and C. krusei $(12 \%)$, which is similar to other studies.[10] Despite report of high mortality for $\mathrm{C}$. tropicalis and C. glabrata (4070\%).[4] in candidemia in VVC mortality is uncommon.

The analysis of the age distribution showed highest frequency of VVC among the 40-49 years age group, which may have a direct correlation with change in the microbiotas a result of hormonal changes in perimenopausal woman.

The highest influence in frequency in the perimenopausal group was seen for C. tropicalis and C. glabrata. C. tropicalis peaked in the 30-39 age group and showed sudden decrease in frequency in the 40-49 age group. C. glabrata peaked in the 40-44 age group and decreased since 45 yrs. Age mark C. albicans was uniformly distributed among all the age groups and no typical pattern of distribution was found for C. krusei. Studies on VVC have generally showed a decreasing trend in post-menopausal women in relation to decreased progesterone and estrogen concentrations, which are known to have suppressive effects on the anti-Candida activity of neutrophils and vaginal 
epithelial cells respectively.[11] Based on the increasing frequency of VVC in perimenopausal women found in this study, it would be interesting to undertake the study in larger population and correlate it with hormonal levels in the study subjects.

Another significant observation was 19\% frequency of isolates from post-operative cases of ectopic pregnancy. In a recent randomised controlled trial, 4000 asymptomatic pregnant women were screened for bacterial vaginosis, candidiasis and/or trichomoniasis during early pregnancy (15-19 weeks' gestation) followed by treatment against the respective infections reduced the spontaneous preterm birth rate by $46 \%{ }^{[12]}$ Such type of studies involving larger population of pregnant women may also help in reducing the incidence of preterm birth as well as help in establishing the cause and effect relationship of VVC among ectopic pregnancy cases.

Among the azoles, the highest susceptibility was seen towards fluconazole followed by voriconazole and the least was towards ketoconazole. Exceptions were C. glabrata isolates which showed higher susceptibility towards voriconazole $(83 \%)$ as compared to fluconazole $(75 \%)$. All isolates of C. albicans were sensitive to fluconazole similar to studies showing isolation of fluconazole resistant $\mathrm{C}$. albicans in VVC to be a rare event, although certain amount of resistance may appear due to prolonged therapy of recurrent candidiasis.[13]

NAC spp. are known to be inherently or secondarily resistant to fluconazole.[4] However, through our study only $14 \%$ of C. krusei, $25 \%$ of C. glabrata and $20 \%$ of C. tropicalis were found to be resistant from the present study; hence, it may be inferred that fluconazole is the most efficient of the azoles for treating the local VVC cases other than candidiasis caused by C. glabrata, in which case voriconazole is advised.

Among the fluconazole resistant isolates, most were susceptible to voriconazole, similar to studies reporting that more than 95\% fluconazole resistant isolates of C. albicans, C. glabrata and $\mathrm{C}$. krusei are sensitive to voriconazole, though at high MIC values.[14] Absence of any fluconazole resistant isolate of C. albicans and fewer numbers of isolates of $\mathrm{C}$. glabrata and C. krusei was a limitation of the study. All fluconazole resistant C. tropicalis isolates were, however, resistant to voriconazole and ketoconazole.

Resistance to amphotericin B has rarely been observed until recently.[15] There are difficulties in determining the susceptibility breakpoints for amphotericin B and according to currently set limits a minority of in-vitro sensitive isolates have reported clinical resistance. As per current trends, C. albicans and C. parapsilosis are known to be fully susceptible to amphotericin B, while C. glabrata has high MICs and C. tropicalis, C. krusei and C. guilliermondii are known to be susceptible with occasional resistance.[16]

In the present study, most species were sensitive to amphotericin B, although C. glabrata showed higher MIC values. Exception towards polyene sensitivity was seen for 6 (40\%) C. tropicalis isolates. Whether the particular isolates were from recurrent VVC cases and have acquired resistance, clinical resistance developed over the years through treatment or are intrinsically resistant is a subject of further study and is essential for determining the treatment for VVC among the local population.

To summarise, incidence of VVC among the hospital attending patients was found to be $25 \%$. NAC spp. (62\%) prevailed over C. albicans (38\%) with C. tropicalis occurring most commonly among the NAC spp. All the drugs apart from ketoconazole showed good efficacy. Among the azoles, fluconazole was found more effective for treatment of $\mathrm{C}$. albicans, C. tropicalis and C. krusei over voriconazole and ketoconazole. Voriconazole though known to have broad spectrum activity was effective over fluconazole only for C. glabrata isolates. Polyenes were found to be effective for all species except $C$. tropicalis.

Whether antifungal resistance profile varied for recurrent VVC cases in the local population should be looked into in future studies. Surveillance and monitoring the resistance pattern of VVC isolates are essential in order to reduce the complication among the subjects of reproductive age group. Molecular characterization of the isolates as well as determination of drug-resistant mutants would further strengthen the aforesaid program.

ACKNOWLEDGEMENT : The authors thank the Department of Biotechnology under Ministry of Science and Technology, India for funding the study and Dr. Sumathi Muralidhar, Associate Professor \& Senior Microbiologist, Apex-Regional STD Teaching, Training \& Research Centre, Vardhman Mahavir Medical College and Safdarjang Hospital, New Delhi for kindly providing with the reference strains of C. albicans

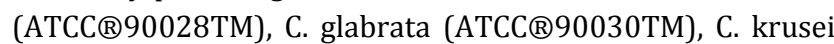
(ATCC $® 6258 T M)$ C. tropicalis (ATCC®750TM) and C. parapsilosis (ATCC®22019TM). The authors also thank Dr. KVR Reddy, Deputy Director (Sr. Grade) \& Head, Division of Molecular Immunology \& Microbiology (MIM), National Institute for Research in Reproductive Health (NIRRH), Mumbai for his scientific advice and guidance.

\section{REFERENCES:}

1. Egan ME, Lipsky MS. Diagnosis of vaginitis. Am Fam Physician 2000; 62(5):1095-104.

2. Anderson MR, Klink K, and Cohrssen A. Evaluation of vaginal complaints. JAMA 2004; 291(11):1368-79.

3. Bauters TG, Dhont MA, Temmerman MI, Nelis HJ. Prevalence of vulvovaginal candidiasis and susceptibility to fluconazole in women. Am J Obstet Gynecol 2002; 187(3):569-74.

4. Krcmery V, Barnes AJ. Non-albicansCandida spp. causing fungaemia: Pathogenicity and antifungal resistance. J Hosp Infect 2002; 50(4):243-60.

5. Nusbaum MR, Wallace RR, Slatt LM, Kondrad EC. Sexually transmitted infections and increased risk of coinfection with human immunodeficiency virus. J Am Osteopath Assoc 2004; 104(12):527-35.

6. Saikia L, Nath R, Deuori T, Mahanta J. Sexually transmitted diseases in Assam: An experience in a tertiary care referral hospital. Indian J Dermatol Venereol Leprol 2009; 75(3):329. 
7. Clinical and Laboratory Standards Institute. Method for Antifungal Disk Diffusion Susceptibility Testing of Yeasts; Approved Guidelines-Second edition Vol.29 No.17, August-2009 CLSI document M44-A2.

8. Mohanty S, Xess I, Hasan F, Kapil A, Mittal S, Tolosa JE. Prevalence \& susceptibility to fluconazole of Candida species causing vulvovaginitis. Indian J Med Res 2007; 126(3):216-19.

9. Sardi JC, Scorzoni L, Bernardi T, Fusco-Almeida AM, Mendes Giannini MJ. Candidaspecies: Current epidemiology, pathogenicity, biofilm formation, natural antifungal products and new therapeutic options. J Med Microbiol 2013; 62(1):10-24.

10. Parazzini F, Di Cintio E, Chiantera V, Guaschino S. Determinants of different Candida species infections of the genital tract in women. Sporachrom Study Group. Eur J Obstet Gynecol Reprod Biol 2000; 93(2):141-45.

11. Omar AA. Gram stain versus culture in the diagnosis of vulvovaginal candidiasis. East Mediterr Health J 2001; 7(6):925-34.

12. Kiss H, Petricevic L, Husslein P. Prospective randomised controlled trial of an infection screening programme to reduce the rate of preterm delivery. BMJ 2004; 329(7462):371.

13. Sobel JD, Wiesenfeld HC, Martens M, Danna P, Hooton TM, Rompalo A et al. Maintenance fluconazole therapy for recurrent vulvovaginal candidiasis. N. Engl J Med 2004; 351(9):876-83.

14. Ruhnke M, Schmidt-Westhausen A, Trautmann M. Invitro activities of voriconazole (UK-109,496) against fluconazole-susceptible and resistant Candida albicans isolates from oral cavities of patients with human immunodeficiency virus infection. Antimicrob Agents Chemother 1997; 41: 575-77.

15. Krogh-Madsen M, Arendrup MC, Heslet L, Knudsen JD. Amphotericin B and caspofungin resistance in Candida glabrata isolates recovered from a critically ill patient. Clin Infect Dis 2006; 42(7): 938-44.

16. David E. Amphotericin B: Spectrum and resistance. J Antimicrob Chemother 2002; 49 (S1): 7-10.

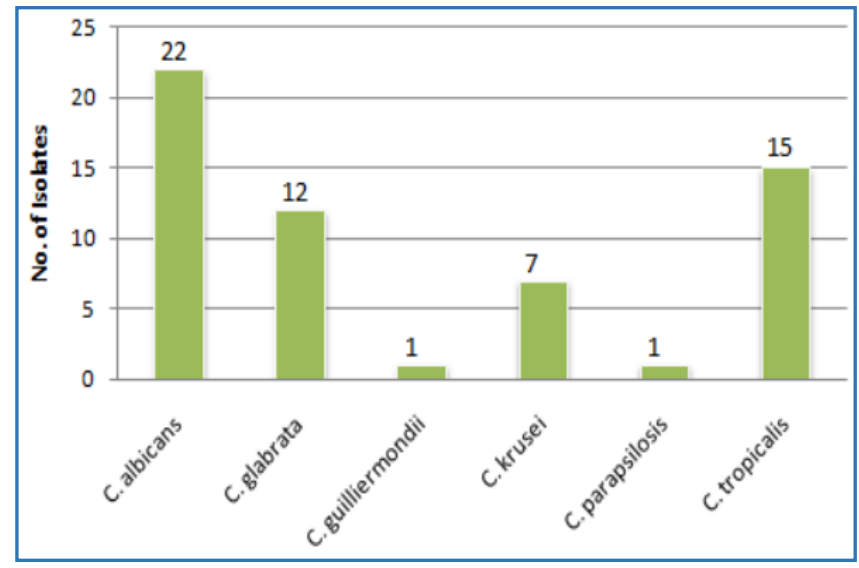

Fig 1: Frequency of various Candida Species Isolated in the study

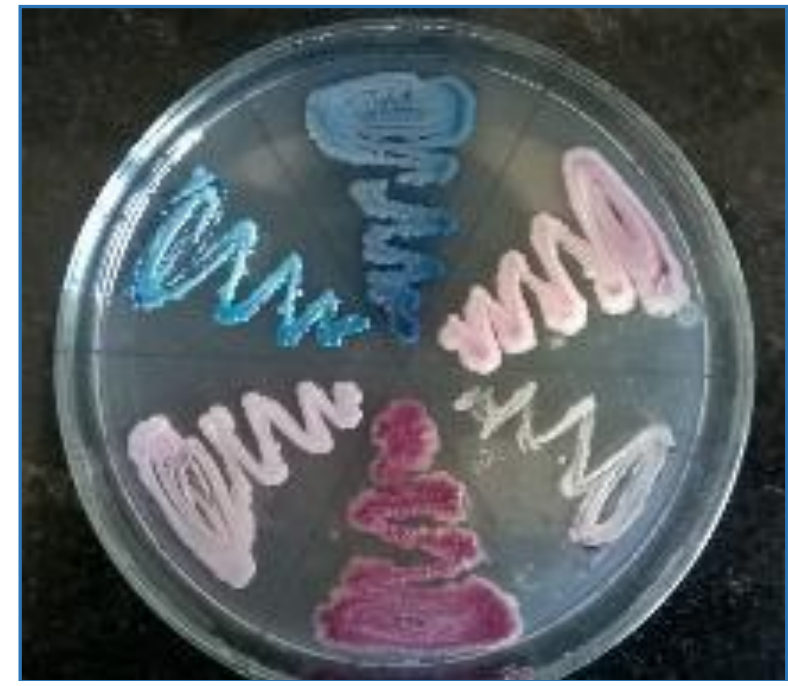

Fig 2: Speciation of Candida on HiCrome agar. Clockwise from top: C. tropicalis, C. glabrata, C. guilliermondii, $C$. krusei, C. parapsilosis and C. albicans

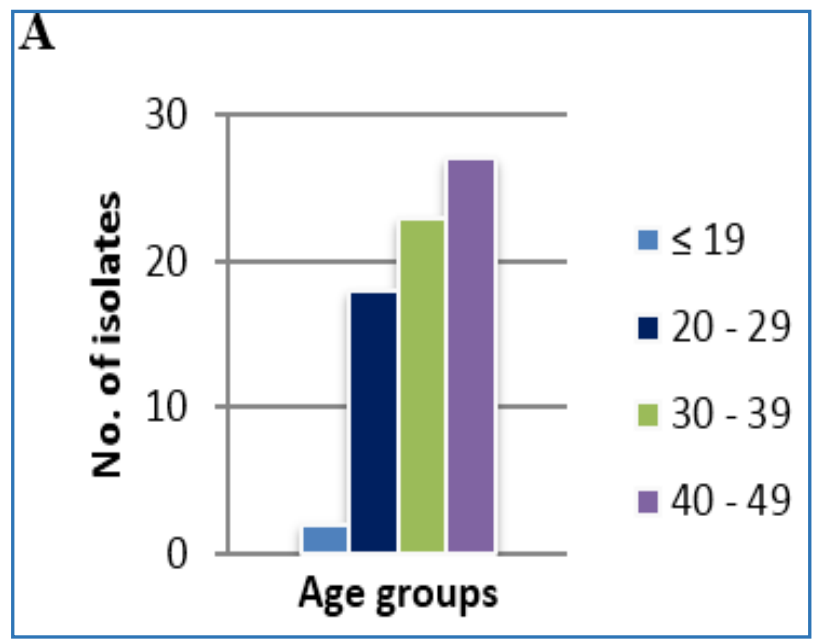

Fig 3: A. Incidence of VVC among various age groups including the isolates that could not be isolated by culture

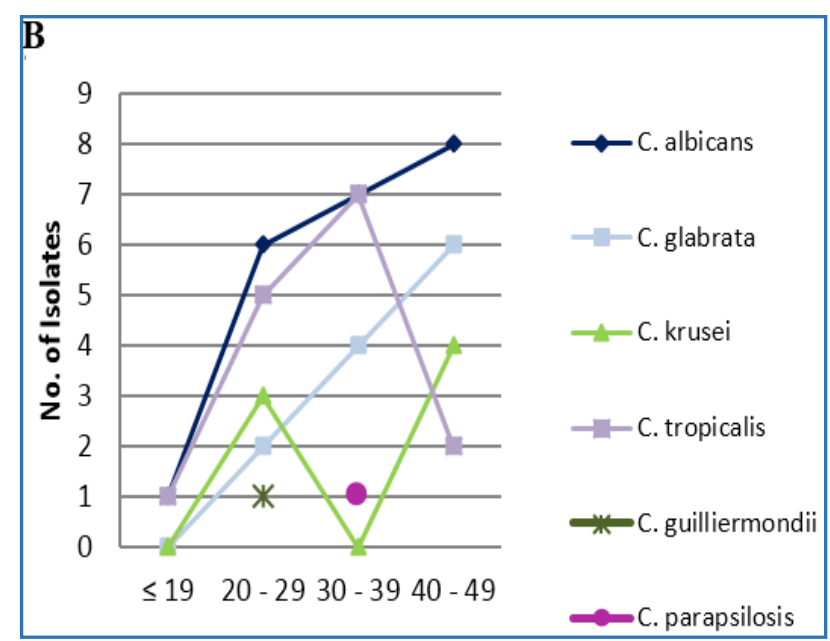

Fig 3: B. Species wise incidence of VVC among various age groups. The graphical representation could be done for only the 58 isolates that could be isolated by culture and hence speciated 


\begin{tabular}{|c|c|c|c|c|}
\hline & Polyenes & \multicolumn{3}{|c|}{ Azoles } \\
\hline & & \multicolumn{2}{|c|}{$\begin{array}{c}\text { Triazole } \\
\text { based }\end{array}$} & $\begin{array}{c}\text { Imidazole } \\
\text { based }\end{array}$ \\
\hline & Amphotericin B & Fluconazole & Voriconazole & Ketoconazole \\
\hline C. albicans(n =22) & $20(91)$ & $22(100)$ & $20(91)$ & $18(82)$ \\
\hline C. glabrata(n = 12) & $10(83)$ & $9(75)$ & $10(83)$ & 0 \\
\hline C. guilliermondii(n = 1) & $1(100)$ & $1(100)$ & $1(100)$ & $1(100)$ \\
\hline C. krusei(n = 7) & $7(100)$ & $6(86)$ & $5(71)$ & $1(14)$ \\
\hline C. parapsilosis(n = 1) & 0 & $1(100)$ & $1(100)$ & 0 \\
\hline C. tropicalis(n = 15) & $9(60)$ & $12(80)$ & $12(80)$ & $10(67)$ \\
\hline All isolates (n = 58) & $47(81)$ & $51(88)$ & $49(84)$ & $30(52)$ \\
\hline \multicolumn{2}{|c|}{ Table 1: Antifungal Susceptibility Testing. Numbers in } \\
Parenthesis represent Percentage of Susceptibility. \\
\hline
\end{tabular}

